\title{
Bacillus Calmette-Guérin (BCG) Treatment Failures with Non-Muscle Invasive Bladder Cancer: A Data-Driven Definition for BCG Unresponsive Disease
}

\author{
Ryan L. Steinberg ${ }^{\mathrm{a}}$, Lewis J. Thomas ${ }^{\mathrm{a}}$, Sarah L. Mott ${ }^{\mathrm{b}}$ and Michael A. O’Donnell ${ }^{\mathrm{a}, \mathrm{b}, *}$ \\ ${ }^{a}$ University of Iowa Department of Urology, Iowa City, IA, USA \\ ${ }^{\mathrm{b}}$ University of Iowa Holden Comprehensive Cancer Center, Iowa City, IA, USA
}

\begin{abstract}
.
Objective: To create the first data-driven definition for those unlikely to benefit from further BCG treatment.

Materials and Methods: The database created for the Phase 2 BCG-Interferon- $\alpha$ 2B (IFN) study was queried and BCG failure patients were identified $(n=334)$. Full study protocols have previously been published. Separate models were constructed for analysis of patients with any CIS (pure or concomitant) and pure papillary disease. Variables considered included age, gender, stage, grade, tumor size and focality (for papillary only), number of prior BCG courses, and prior BCG failure interval.

Results: Patients with recurrent CIS within 6 months of their most recent prior BCG course (HR 2.56, $p<0.01$ ) and $\geq 2$ prior BCG failures (HR 1.54, $p<0.01$ ) responded worst to repeat intravesical therapy. Those with CIS recurrence at 6-12 months did not differ from those recurring within 6 months $(\mathrm{HR}=0.88, p=0.71)$. Patients with recurrent papillary disease within 6 months (HR 1.82, $p=0.02$ ), $\geq 2$ BCG failures (HR 1.54, $p=0.03$ ), and multifocal disease (HR 2.05, $p<0.01$ ) responded worst to therapy. Patients with T1 disease remained disease free in 38\% of cases (24-51\% 95\% CI) at 2 years with low rates of progression.

Conclusions: Patients who fail two courses of BCG with either persistent or recurrent multifocal papillary disease within 6 months or CIS within 12 months of their prior BCG should be considered BCG unresponsive. Recurrent T1 disease respond reasonably well to another course with low progression rates but further investigation is warranted.
\end{abstract}

Keywords: Urinary bladder neoplasms, mycobacterium bovis, treatment failure

\section{INTRODUCTION}

Intravesical Bacillus Calmette-Guérin (BCG) remains the standard of care after transurethral resection for high-grade and recurrent low-grade non-muscle invasive bladder cancer (NMIBC) with clear superiority over chemotherapy [1-5]. Unfortunately, initial BCG therapy fails in approximately $40 \%$ of patients over a 2-year span [6]. Cystectomy

\footnotetext{
*Correspondence to: Michael A. O'Donnell, MD, University of Iowa, Department of Urology, 200 Hawkins Dr., 3231 RCP, Iowa City, IA 52242-1089, USA. Tel.: +1 319353 8939; Fax: +1 319 356 3900; E-mail: michael-odonnell@uiowa.edu.
}

remains the preferred treatment for high risk recurrent disease per the American Urological Association (AUA) [7] and European Association of Urology (EAU) guidelines [8], but radical surgery comes with substantial risk [9] and dramatic lifestyle changes. Further, many patients are unwilling to accept major surgery until all other possibilities are exhausted. Additional intravesical therapy is another treatment option but risks the possibility of disease progression and/or metastasis during retreatment. The most utilized agent is a second course of intravesical BCG, but this is only effective in $35 \%$ of cases $[10,11]$. 
Prior to 2005, the definition of "BCG refractory" was surgeon-dependent, anecdotal, and wide ranging [12-14]. The primary consideration in the majority of these definitions was the number of prior BCG courses, while recurrence timing has only been addressed by a few authors. Herr et al. demonstrated that the inability to remain tumor-free by 6 months after BCG induction correlated with both an increase in tumor recurrence and progression, but this was in BCG-naïve patients who received only a single induction BCG course without maintenance [15].

Given the ambiguity, an international consensus panel was assembled in 2005 and created classifications to better describe failure patients [16]. The resulting definition of BCG refractory was "nonimproving or worsening disease at 3 months after initial BCG" or "failure to achieve a disease-free state by 6 months after initial BCG with either maintenance or retreatment." This definition improved the description of BCG failure patients but its use remained inconsistent. More recently, a panel of bladder cancer experts was convened at the 2015 Genitourinary Cancers Symposium of the American Society of Clinical Oncologists. They agreed that a broader functional classification of patients who were unlikely to benefit significantly from additional BCG should be created and the term "BCG unresponsive" was conceived [17].

To our knowledge, there have been no analyses that have assessed what truly constitutes BCG unresponsive disease in a contemporary cohort of BCG failure patients. In this study, we aimed to create a data-driven definition for BCG unresponsive disease.

\section{MATERIALS AND METHODS}

\section{Study population/baseline characteristics}

Institutional Review Board approval was obtained. The large database created as part of the Phase 2 BCG-Interferon- $\alpha$ 2B (IFN) study was queried. Enrollment for this study was liberal and included patients with primary and recurrent NMIBC, with and without carcinoma in situ (CIS), and those with prior chemotherapy and/or BCG treatment. Baseline characteristics have previously been reported [18]. The number of prior BCG courses received was collected. A BCG course was considered to be either an induction course or a complete 3-week maintenance course. Patients who received known ineffective maintenance therapy, i.e. single instillation monthly or single instillation quarterly BCG, were not considered to have received an additional course of BCG therapy. Only $36 \%$ of patients enrolled received prior maintenance therapy and of these, only approximately one third received SWOG protocol maintenance therapy. Thus, the vast majority of patients who received multiple prior BCG courses were treated with repeat induction courses.

\section{Study treatment}

As previously reported, treatment commenced 3-8 weeks after either transurethral resection of bladder tumor (TURBT) or confirmatory cystoscopy, biopsy, or positive cytology. BCG failure patients were administered 1/3 standard dose of either TICE (Organon Teknika, Roseland, NJ) or Connaught (Aventis-Pasteur, Swiftwater, PA) strain BCG per physician preference mixed directly with 50 million units (MU) IFN (Intron A, Schering-Plough, Kenilworth, NJ). Patients who experienced toxicity during induction were moved onto a dose-reduced protocol (66\% reduction with identical timing) after a 2 -week rest period. Additional 2-week treatment delays were allowed for repeat episodes of intolerance, as long as the entire induction cycle was completed within 10 weeks of initiation. All patients without further recurrence then received reduced dose maintenance therapy consisting of 3 "mini cycles" ( 3 weekly BCG instillations) at 3, 9, and 15 months after the end of the induction cycle. Patients began bladder surveillance 4-6 weeks after induction and had repeat assessments every 3 months for 2 years.

\section{Analysis}

Given that patients with CIS have an increased rate of recurrence, progression, and mortality, as well as differences in genetic alterations between CIS (loss of p53) and papillary disease (cyclin D activation) [19], CIS and papillary disease may be considered different disease entities. As such, we elected to create separate statistical models to analyze each group. Cox proportional hazards regression was used to assess the effects of demographic and clinicopathologic variables on treatment failure. Tumor recurrence was defined as: visible tumor, unless histologically confirmed to be benign; definitive positive urine cytology; positive biopsy, even with negative cystoscopy; and any transitional cell carcinoma, regardless of presenting location, including upper 
tract, prostate, urethra, or metastatic. Patients without evidence of a recurrence were censored at date of last contact. Recurrence intervals in both models were indexed from the most recent prior BCG course completed by the patient. Separate multivariable models were derived for patients with any CIS (pure or concomitant) or pure papillary diagnoses. A stepwise variable selection process was used to identify the significant factors among all those under consideration in the multivariable models. A 5\% level of significance was specified for the stepwise selection process. Regression estimates are reported as hazard ratios (HR) and $95 \%$ confidence intervals (CI). Using the Kaplan-Meier method, plot of survival curves stratified by variables identified upon multivariable were constructed. Survival estimates and $95 \%$ pointwise confidence intervals were reported. All statistical testing was two-sided and assessed for significance at the 5\% level using SAS v9.4 (SAS Institute, Cary, NC) statistical software.

\section{RESULTS}

\section{Patient characteristics/univariate analysis}

Three hundred thirty-four patients were identified as having at least 1 prior BCG failure, of which 98 had CIS (pure or concomitant) and 236 patients had pure papillary disease (Table 1). No patients had a history of prior BCG intolerance. Over $70 \%$ of patients in both groups had recurrence within 1 year of prior BCG and approximately $40 \%$ had previously failed 2 or more courses of BCG.

Overall, BCG failure patient characteristics have been previously reported [18]. Univariate analysis identified non-significant differences in outcomes between pure CIS and papillary + CIS $(p=0.66)$ in the CIS model, as well as Ta vs. T1 $(p=0.45)$ and tumor size $(p=0.06)$ in the papillary model (Table 2$)$. In both models, there was no statistically significant difference between those who were never disease-free and those with recurrence within 6 month ( $p=0.06$ in CIS model, $p=0.68$ in papillary model). Over $90 \%$ of patients who failed BCG/IFN were found to have a bladder recurrence.

\section{Multivariable analysis of CIS patients}

Multivariable analysis of CIS patients revealed that the number of prior BCG courses and prior
Table 1

Baseline characteristics of patients with prior BCG failure that received treatment with intravesical BCG/IFN for a NMIBC recurrence. $\mathrm{NS}=$ not specified

\begin{tabular}{|c|c|c|}
\hline & \multicolumn{2}{|c|}{ Disease Type } \\
\hline & $\begin{array}{c}\text { Any CIS } \\
N=98\end{array}$ & $\begin{array}{c}\text { Pure Papillary } \\
\quad N=236\end{array}$ \\
\hline \multicolumn{3}{|l|}{ Gender } \\
\hline Male & $79(81.4 \%)$ & $161(68.2 \%)$ \\
\hline Female & $18(18.6 \%)$ & $75(31.8 \%)$ \\
\hline \multicolumn{3}{|l|}{ Ethnicity } \\
\hline White, Non-Hispanic & $93(96.9 \%)$ & $223(96.5 \%)$ \\
\hline White, Hispanic & $0(0 \%)$ & $3(1.3 \%)$ \\
\hline African American & $3(3.1 \%)$ & $5(2.2 \%)$ \\
\hline Median Age (Years (Range)) & $72(42-90)$ & $72(41-93)$ \\
\hline \multicolumn{3}{|l|}{ Stage } \\
\hline CIS alone & $70(71.4 \%)$ & $0(0 \%)$ \\
\hline Papillary + CIS & $28(28.6 \%)$ & $0(0 \%)$ \\
\hline $\mathrm{Ta}$ & $16(16.3 \%)$ & $0(0 \%)$ \\
\hline $\mathrm{T} 1$ & $10(10.2 \%)$ & $0(0 \%)$ \\
\hline $\mathrm{Ta}+\mathrm{T} 1$ & $2(2.1 \%)$ & $0(0 \%)$ \\
\hline Papillary & $0(0 \%)$ & $236(100 \%)$ \\
\hline $\mathrm{Ta}$ & $0(0 \%)$ & $175(74.2 \%)$ \\
\hline $\mathrm{T} 1$ & $0(0 \%)$ & $56(23.7 \%)$ \\
\hline $\mathrm{Ta}+\mathrm{T} 1$ & $0(0 \%)$ & $5(2.1 \%)$ \\
\hline \multicolumn{3}{|l|}{ Grade } \\
\hline Low & $0(0 \%)$ & $72(32.7 \%)$ \\
\hline Intermediate & $0(0 \%)$ & $91(41.3 \%)$ \\
\hline High & $98(100 \%)$ & $57(25.9 \%)$ \\
\hline \multicolumn{3}{|l|}{ Tumor Size } \\
\hline$<1 \mathrm{~cm}$ & NS & $101(47.9 \%)$ \\
\hline$\geq 1 \mathrm{~cm}$ & NS & $110(52.1 \%)$ \\
\hline \multicolumn{3}{|l|}{ Number of Tumors } \\
\hline Solitary & NS & $69(30.7 \%)$ \\
\hline$>1$ Tumor & NS & $156(69.3 \%)$ \\
\hline \multicolumn{3}{|l|}{ Prior BCG Courses } \\
\hline$\leq 1 \mathrm{BCG}$ failure & $55(56.1 \%)$ & $143(60.6 \%)$ \\
\hline$\geq 2$ BCG failures & $43(43.9 \%)$ & $93(39.4 \%)$ \\
\hline \multicolumn{3}{|l|}{ Prior BCG Failure Interval } \\
\hline$<6$ Months & $47(48.0 \%)$ & $115(48.7 \%)$ \\
\hline 6-12 Months & $23(23.4 \%)$ & $69(29.3 \%)$ \\
\hline$>12$ Months & $28(28.6 \%)$ & $52(22.0 \%)$ \\
\hline Treatment Failure & $52(53.0 \%)$ & $124(52.5 \%)$ \\
\hline Median Time to Failure (Months) & 15 & 21 \\
\hline
\end{tabular}

BCG failure interval were significantly associated with BCG/IFN treatment failure (Table 3a). Patients with 2 or more prior courses of BCG were at increased risk of failure compared to those with less than 2 prior failures $(\mathrm{HR}=2.74, p<0.01)$. Patients who recurred within 6 months $(\mathrm{HR}=2.59$, $p<0.01)$ and at $6-12$ months $(\mathrm{HR}=2.29, p=0.04)$ from their most recent prior BCG induction course were at increased risk of failure as compared to those recurring beyond 12 months. Given the similarity in outcomes and for the purposes of constructing recurrence curves, the $<6$ months and 6-12 month cohorts were combined into a $<12$ month group. Patients with recurrence within 12 
Table 2

Univariate analysis of disease-related variables and the associated risk of treatment failure in BCG failure patients with (A) any recurrent CIS or (B) recurrent pure papillary disease treated with intravesical BCG/IFN

\begin{tabular}{|c|c|c|c|c|c|c|}
\hline \multicolumn{7}{|l|}{$\overline{(A)}$} \\
\hline Covariate & & Level & $\mathrm{N}$ & HR & $95 \% \mathrm{CI}$ & $P$-value \\
\hline \multirow[t]{2}{*}{ Gender } & Male & 79 & 1.12 & 0.53 & 2.39 & 0.76 \\
\hline & Female & 18 & Ref & - & - & - \\
\hline \multirow{2}{*}{ Num Prior TURBTs } & $>5$ & 20 & 1.67 & 0.87 & 3.21 & 0.12 \\
\hline & $1-5$ & 78 & Ref & - & - & - \\
\hline \multirow[t]{2}{*}{ Time Since Index Tumor } & $>2$ years & 28 & 1.36 & 0.73 & 2.51 & 0.33 \\
\hline & $0-2$ years & 59 & Ref & - & - & - \\
\hline \multirow[t]{2}{*}{ Num of Prior BCG Courses } & $2+$ prior courses & 43 & 2.35 & 1.34 & 4.11 & $<.01$ \\
\hline & Less than 1 or 1 prior & 55 & Ref & - & - & - \\
\hline \multirow[t]{2}{*}{ Tumor Size } & $\geq 1 \mathrm{~cm}$ & 24 & 0.80 & 0.38 & 1.66 & 0.54 \\
\hline & $<1 \mathrm{~cm}$ & 37 & Ref & - & - & - \\
\hline \multirow[t]{3}{*}{ Prior BCG Failure Interval } & $<6$ Months & 47 & 2.07 & 1.02 & 4.19 & 0.04 \\
\hline & 6-12 Months & 23 & 2.02 & 0.91 & 4.46 & 0.08 \\
\hline & >12 Months & 28 & Ref & - & - & - \\
\hline \multirow[t]{2}{*}{ BCG Strain } & Connaught & 27 & 1.31 & 0.71 & 2.41 & 0.39 \\
\hline & Tice & 67 & Ref & - & - & - \\
\hline \multirow{2}{*}{ CIS } & Combo & 28 & 0.87 & 0.47 & 1.61 & 0.66 \\
\hline & Pure & 70 & Ref & - & - & - \\
\hline \multicolumn{2}{|l|}{ Age At Study Entry } & 98 & 1.01 & 0.98 & 1.03 & 0.73 \\
\hline \multicolumn{7}{|l|}{ (B) } \\
\hline \multicolumn{2}{|l|}{ Variable } & Level & $\mathrm{N}$ & HR & $95 \% \mathrm{CI}$ & $P$-value \\
\hline \multirow[t]{2}{*}{ Gender } & Male & 161 & 1.01 & 0.70 & 1.46 & 0.96 \\
\hline & Female & 75 & Ref & - & - & - \\
\hline \multirow[t]{2}{*}{ Num Prior TURBTs } & $>5$ & 74 & 1.08 & 0.75 & 1.57 & 0.67 \\
\hline & $1-5$ & 162 & Ref & - & - & - \\
\hline \multirow[t]{2}{*}{ Time Since Index Tumor } & $>2$ years & 89 & 1.13 & 0.78 & 1.63 & 0.52 \\
\hline & $0-2$ years & 130 & Ref & - & - & - \\
\hline \multirow[t]{2}{*}{ Num of Prior BCG Courses } & $2+$ prior courses & 93 & 1.23 & 0.87 & 1.76 & 0.24 \\
\hline & Less than 1 or 1 prior & 143 & Ref & - & - & - \\
\hline \multirow[t]{3}{*}{ Grade } & Intermediate & 91 & 1.10 & 0.73 & 1.68 & 0.64 \\
\hline & High & 57 & 0.90 & 0.55 & 1.48 & 0.69 \\
\hline & Low & 72 & Ref & - & - & - \\
\hline \multirow{2}{*}{ Multifocality } & $>1$ Tumor & 156 & 2.04 & 1.31 & 3.18 & $<.01$ \\
\hline & Solitary Tumor & 69 & Ref & - & - & - \\
\hline \multirow[t]{2}{*}{ Tumor Size } & $\geq 1 \mathrm{~cm}$ & 110 & 1.45 & 0.99 & 2.12 & 0.06 \\
\hline & $<1 \mathrm{~cm}$ & 101 & Ref & - & - & - \\
\hline \multirow[t]{3}{*}{ Prior BCG Failure Interval } & $<6$ Months & 115 & 1.88 & 1.15 & 3.06 & 0.01 \\
\hline & 6-12 Months & 69 & 1.20 & 0.69 & 2.08 & 0.51 \\
\hline & $>12$ Months & 52 & Ref & - & - & - \\
\hline \multirow[t]{2}{*}{ BCG Strain } & Connaught & 71 & 0.78 & 0.52 & 1.17 & 0.23 \\
\hline & Tice & 147 & Ref & - & - & - \\
\hline \multirow[t]{2}{*}{ Tumor Stage } & $\mathrm{T} 1$ & 60 & 1.17 & 0.78 & 1.74 & 0.45 \\
\hline & $\mathrm{Ta}$ & 175 & Ref & - & - & - \\
\hline Age At Study Entry & & 236 & 1.01 & 0.99 & 1.03 & 0.21 \\
\hline
\end{tabular}

months and $\geq 2$ BCG failures performed the worst with only $21 \%(8-38 \% 95 \% \mathrm{CI})$ disease-free at 1 year and $14 \%(3-32 \%)$ disease-free at 2 years (Fig. 1).

\section{Multivariable analysis of pure papillary patients}

Like CIS patients, multivariable analysis of patients with pure papillary disease demonstrated that the number of prior BCG courses and prior BCG failure interval were associated with treatment failure (Table $3 b$ ). Papillary patients with $\geq 2$ prior BCG courses were more likely to fail BCG/IFN than those with less than 2 prior courses $(\mathrm{HR}=1.54, p=0.03)$. Similarly, patients with multifocal disease were more likely to fail treatment than those with a solitary tumor $(\mathrm{HR}=2.05, p<0.01)$. Patients with recurrence within 6 months of their most recent prior BCG induc- 
Table 3

Multivariable analysis of disease-related variables and the associated risk of treatment failure in $\mathrm{BCG}$ failure patients with (A) any recurrent CIS or (B) recurrent pure papillary disease treated with intravesical BCG/ IFN. (N denotes sample size) Note: \# signifies that there was no significant difference between these two groups

\begin{tabular}{|c|c|c|c|c|c|c|}
\hline \multicolumn{7}{|l|}{ (A) } \\
\hline & \multirow[b]{2}{*}{ Level } & \multirow[b]{2}{*}{$\mathrm{N}$} & \multicolumn{4}{|c|}{ Treatment Failure } \\
\hline & & & HR & & & $P$-value \\
\hline \multirow[t]{2}{*}{ Number of Prior BCG Courses } & $\geq 2$ courses & 43 & 2.74 & 1.54 & 4.86 & $<.01$ \\
\hline & $\leq 1$ courses & 55 & Ref & - & - & - \\
\hline \multirow[t]{3}{*}{ Prior BCG Failure Interval } & $<6$ Months & 47 & 2.59 & 1.26 & 5.35 & $<.01$ \\
\hline & 6-12 Months & 23 & 2.29 & 1.03 & 5.08 & 0.04 \\
\hline & $>12$ Months & 28 & Ref & - & - & - \\
\hline Total & & 98 & & & & \\
\hline \multicolumn{7}{|l|}{ (B) } \\
\hline & & & \multicolumn{4}{|c|}{ Treatment Failure } \\
\hline & Level & $\mathrm{N}$ & $\overline{\mathrm{HR}}$ & & & $P$-value \\
\hline \multirow[t]{2}{*}{ Number of Prior BCG Courses } & $\geq 2$ courses & 89 & 1.54 & 1.05 & 2.25 & 0.03 \\
\hline & $\leq 1$ courses & 136 & Ref & - & - & - \\
\hline \multirow[t]{2}{*}{ Multifocality } & $>1$ Tumor & 156 & 2.05 & 1.31 & 3.19 & $<.01$ \\
\hline & Solitary Tumor & 69 & Ref & - & - & - \\
\hline \multirow[t]{3}{*}{ Prior BCG Failure Interval } & $<6$ Months & 113 & 1.82 & 1.08 & 3.01 & 0.02 \\
\hline & 6-12 Months & 67 & 1.00 & 0.56 & 1.78 & 1.00 \\
\hline & $>12$ Months & 45 & Ref & - & - & - \\
\hline Total & & 225 & & & & \\
\hline
\end{tabular}

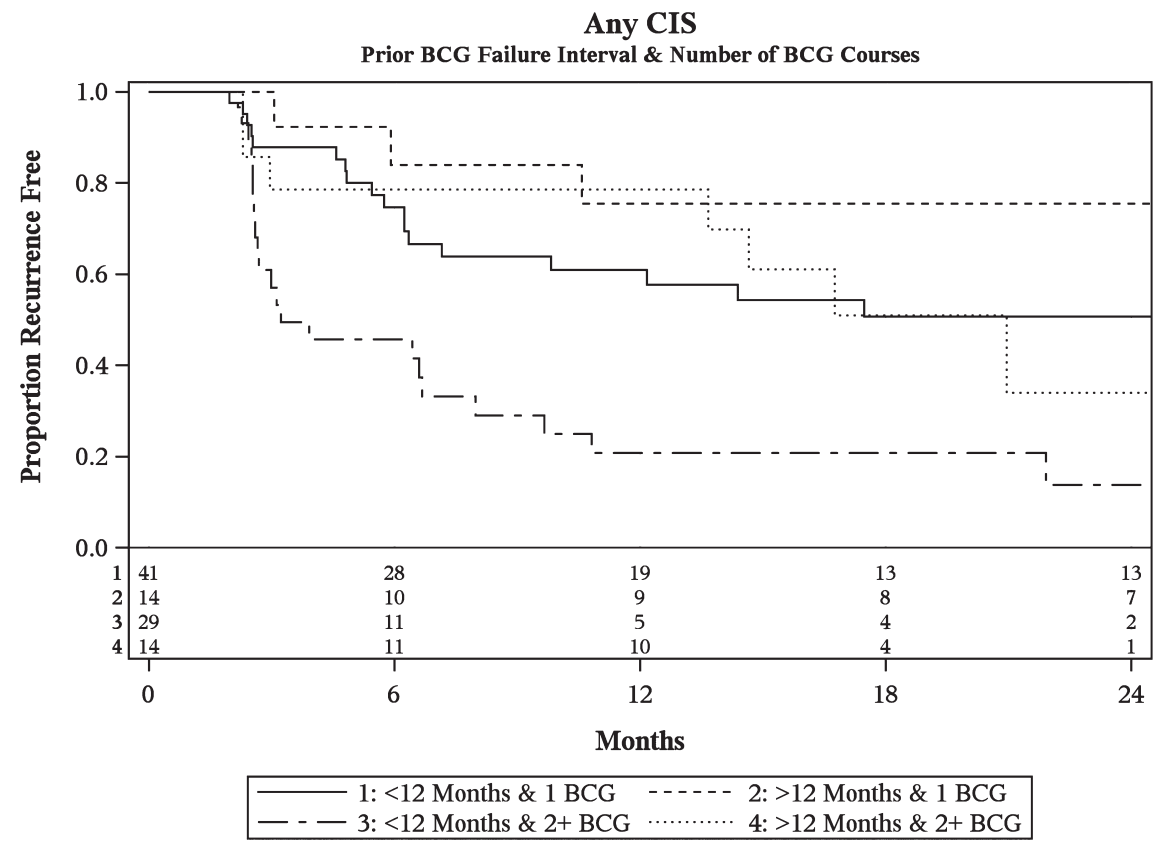

Fig. 1. Kaplan-Meier plot of treatment success of intravesical BCG/IFN in BCG Failure patients with recurrent CIS stratified by prior BCG failure interval and number of prior BCG failures including number at-risk.

tion course were more likely to fail than those at $>12$ months $(\mathrm{HR}=1.82, p=0.02)$. There was no difference appreciated when comparing patients with recurrence between 6-12 months and $>12$ months $(\mathrm{HR}=1.00, p=1.0)$. As such, patients with recur- rence at $6-12$ months and $>12$ months were combined into a $>6$ month group when constructing recurrence curves (Fig. 2).

In addition, multifocal disease was also found to be associated with an increased risk of failure. 
Patients with $\geq 2$ prior BCG courses demonstrated worse outcomes with multifocal disease, while those with $\leq 1$ prior $\mathrm{BCG}$ course demonstrated similar outcomes to estimates considering only recurrence timing and number of prior BCG failures. The estimated recurrence-free rate for a patient with multifocal disease, recurrence within 6 months, and $\geq 2$ prior BCG failures is $20 \%$ (4-44\%) at 2 years, while the same patient with a solitary tumor had a $44 \% 2$ year recurrence-free rate. Though, it should be noted that the latter point estimate is derived from a small subgroup $(n=10)$ with a wide confidence interval $(12-73 \%)$.

\section{Analysis of high grade T1 disease}

Sixty-one patients in our cohort were identified as having previously failed BCG and diagnosed with $\mathrm{T} 1$ disease at the time of study entry. Of these 61 patients, $51 \%(36-63 \%)$ remained disease-free at 1 year and $38 \%(24-51 \%)$ at 2 years after treatment. Further analysis of this group, particularly with respect to recurrence timing, was limited by the sample size. The rate of overall progression for $\mathrm{T} 1$ disease was low $(10 \%)$ in patients with a single prior BCG failure, but became clinically significant after 2 prior BCG failures $(23.6 \%)$ [20]. Table 4 provides a list of expected

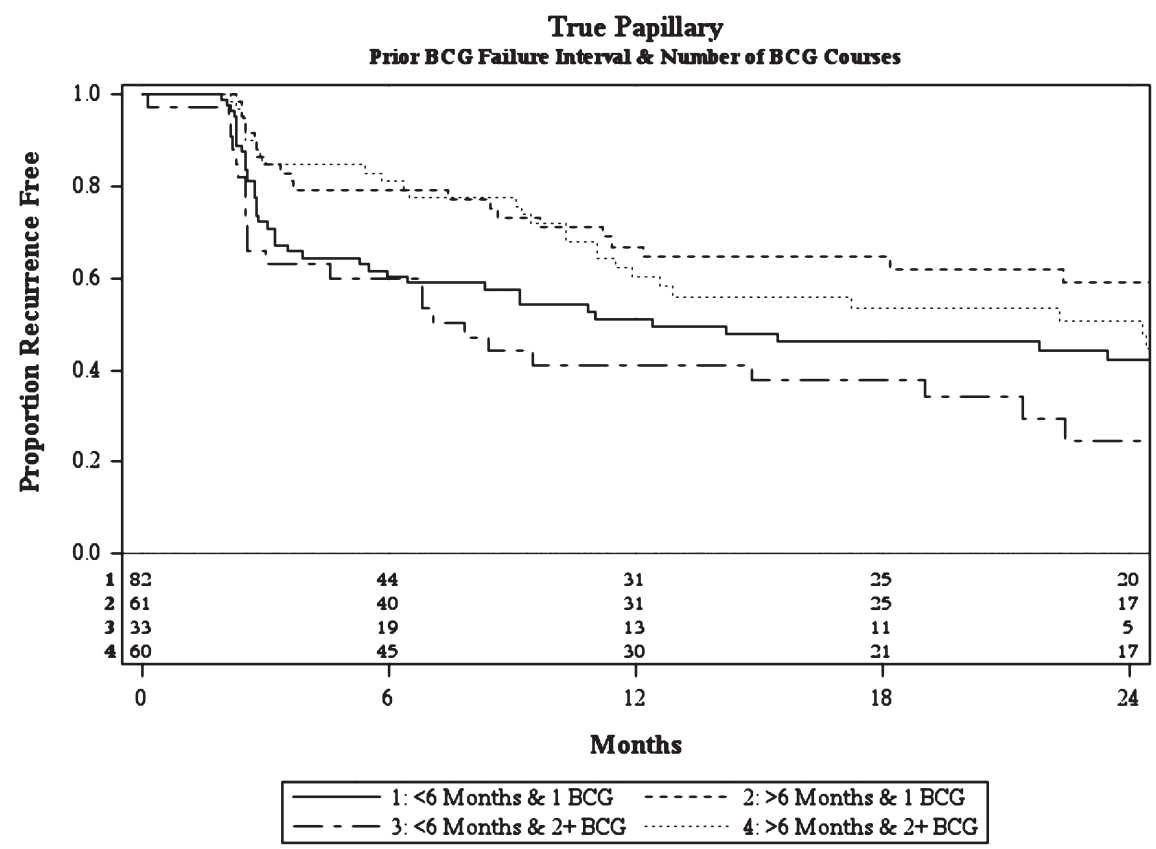

Fig. 2. Kaplan-Meier plot of treatment success of intravesical BCG/IFN in BCG Failure patients with recurrent pure papillary disease stratified by prior BCG failure interval and number of prior BCG failures including number at-risk.

Table 4

Estimated recurrence-free rate of patients with prior BCG failure treated with intravesical BCG/IFN for recurrent NMIBC based upon the prior BCG failure interval and number of prior BCG courses at study enrollment

\begin{tabular}{|c|c|c|c|c|c|c|c|}
\hline Disease Type & $\begin{array}{c}\text { Prior BCG } \\
\text { Failure Interval }\end{array}$ & BCG & Focality & $n$ & 3 Months & 12 Months & 24 Months \\
\hline \multirow[t]{4}{*}{ Any CIS } & $<12$ Months & $\leq 1$ courses & - & 41 & $88 \%(73-95 \%)$ & $61 \%(44-74 \%)$ & $51 \%(33-66 \%)$ \\
\hline & & $\geq 2$ courses & - & 29 & $57 \%(37-73 \%)$ & $21 \%(8-38 \%)$ & $14 \%(3-32 \%)$ \\
\hline & $>12$ Months & $\leq 1$ courses & - & 14 & $100 \%$ & $76 \%(42-91 \%)$ & $76 \%(42-91 \%)$ \\
\hline & & $\geq 2$ courses & - & 14 & $79 \%(47-93 \%)$ & $79 \%(47-93 \%)$ & $34 \%(7-65 \%)$ \\
\hline \multirow[t]{6}{*}{ Pure Papillary } & $<6$ Months & $\leq 1$ courses & All & 82 & $72 \%(61-81 \%)$ & $51 \%(40-62 \%)$ & $42 \%(30-54 \%)$ \\
\hline & & $\geq 2$ courses & All & 33 & $66 \%(47-80 \%)$ & $41 \%(25-57 \%)$ & $25 \%(10-42 \%)$ \\
\hline & & & $>1$ Tumor & 18 & $60 \%(33-78 \%)$ & $30 \%(11-52 \%)$ & $20 \%(4-44 \%)$ \\
\hline & $>6$ Months & $\leq 1$ courses & All & 61 & $85 \%(73-92 \%)$ & $67 \%(52-78 \%)$ & $59 \%(44-72 \%)$ \\
\hline & & $\geq 2$ courses & All & 60 & $85 \%(73-92 \%)$ & $61 \%(46-72 \%)$ & $51 \%(36-64 \%)$ \\
\hline & & & $>1$ Tumor & 33 & $85 \%(67-93 \%)$ & $51 \%(32-67 \%)$ & $40 \%(23-57 \%)$ \\
\hline $\mathrm{T} 1$ & & & & 61 & $80 \%(67-88 \%)$ & $51 \%(36-63 \%)$ & $38 \%(24-51 \%)$ \\
\hline
\end{tabular}


rates of treatment efficacy for this group and all others previously discussed.

\section{DISCUSSION}

Determining that a patient is "BCG unresponsive" is a critical distinction to make in a patient's treatment course so as not to delay delivery of alternative intravesical agents or radical surgery that can confer a better survival advantage. To date, the definition of what constitutes "BCG unresponsive" disease has been either anecdotal or through an expert panel consensus without a direct data-driven analysis.

Our study identified that the number of prior BCG failures and the prior BCG failure interval were common variables predictive of treatment failure in both CIS and pure papillary disease. With respect to prior BCG, our data confirms the previously published reports that patients who fail two or more prior BCG courses respond significantly worse to therapy than those with one prior failure $[10,18]$. With regard to prior BCG failure interval, patients with CIS recurrence within 12 months of prior BCG performed worse than those beyond 12 months. While many definitions of BCG unresponsive disease utilize 6 months as the cutoff, our study supports previously published literature [21] suggesting that a longer interval beyond 6 months is needed, as recurrence at 6-12 months portends just as poor of a response to repeat BCG-based therapy as those with recurrence within 6 months. Conversely, patients with pure papillary disease had a distinctively worse response to therapy when recurrence occurred within 6 months, a time interval more in line with the currently utilized definitions for unresponsive disease.

Tumor multifocality was also identified as an independent risk factor for treatment failure in those with papillary disease. This is in line with other reports that have identified multifocality as a predictor of recurrence and progression [22]. The effect of multifocality on the recurrence-free rate was most apparent in patients with $\geq 2$ prior BCG failures. Given the impact of multifocality on treatment failure, it can be argued that a definition for BCG unresponsive disease that excludes multifocality would be incomplete. However, the full impact of what constitutes multifocal disease, such as the mapping of tumor distribution relative to one another, as well as the specific stage, grade and size of each tumor, remains incompletely evaluated. As previously reported in the study interim analysis [20], overall progression rates (with- out metastasis, with metastasis, and death) were low $(7.8 \%)$, as was the rate of bladder cancer mortality $(0.9 \%)$.

For patients with $\mathrm{T} 1$ disease, we identified a reasonable response to therapy with $38 \%$ (24-51\%) disease-free at 2 years and no significant difference in outcomes when compared to patients with Ta disease. Unfortunately, this cohort was too small for further sub-analysis. The rate of overall progression was low $(10 \%)$ in patients with a single prior BCG failure, but became clinically significant after 2 prior BCG failures (23.6\%). While encouraging, these results should be interpreted cautiously given the small cohort size and short, 24-month median follow up time.

Minimal contemporary data exists on conservative management (repeat TUR and BCG) for recurrent T1 disease after prior BCG [23] and is limited by changes in practice (i.e. lack of maintenance BCG, repeat resection) and the duration of follow-up. In recurrent $\mathrm{T} 1$ disease, even in those with long diseasefree intervals or few prior BCG induction courses, the risk of disease progression, not disease recurrence, may ultimately prove to be the primary consideration in selecting the next therapy, making the inclusion of recurrent $\mathrm{T} 1$ disease in the definition for BCG unresponsive a moot point. Given the small cohort size and limited follow up, a definition for BCG unresponsive disease that includes patients with recurrent $\mathrm{T} 1$ disease cannot be formulated at this time. Further evaluation of these patients is assuredly warranted.

Given the above, we suggest that the following definitions be used to better describe BCG failure patients. "BCG unresponsive" disease should be defined as any patient who fails 2 courses of BCG with either persistent or recurrent multifocal papillary disease within 6 months or persistent or recurrent CIS within 12 months. Our definition closely resembled that published by Nieder et al. [16] with the exception that it allows for a longer prior BCG failure interval in patients with CIS and specifies multifocal disease as a parameter for papillary disease. Patients with recurrent disease who do not meet these criteria should be considered "BCG relapsing." Further investigation of recurrent Ta disease at 6-12 months, the effect of multifocality in specific subpopulations, and $\mathrm{T} 1$ disease is warranted to assess if there is any distinction in outcomes to suggest the presence of an early vs. late relapsing group. Figure 3 provides a proposed treatment algorithm for patients with NMI disease. 


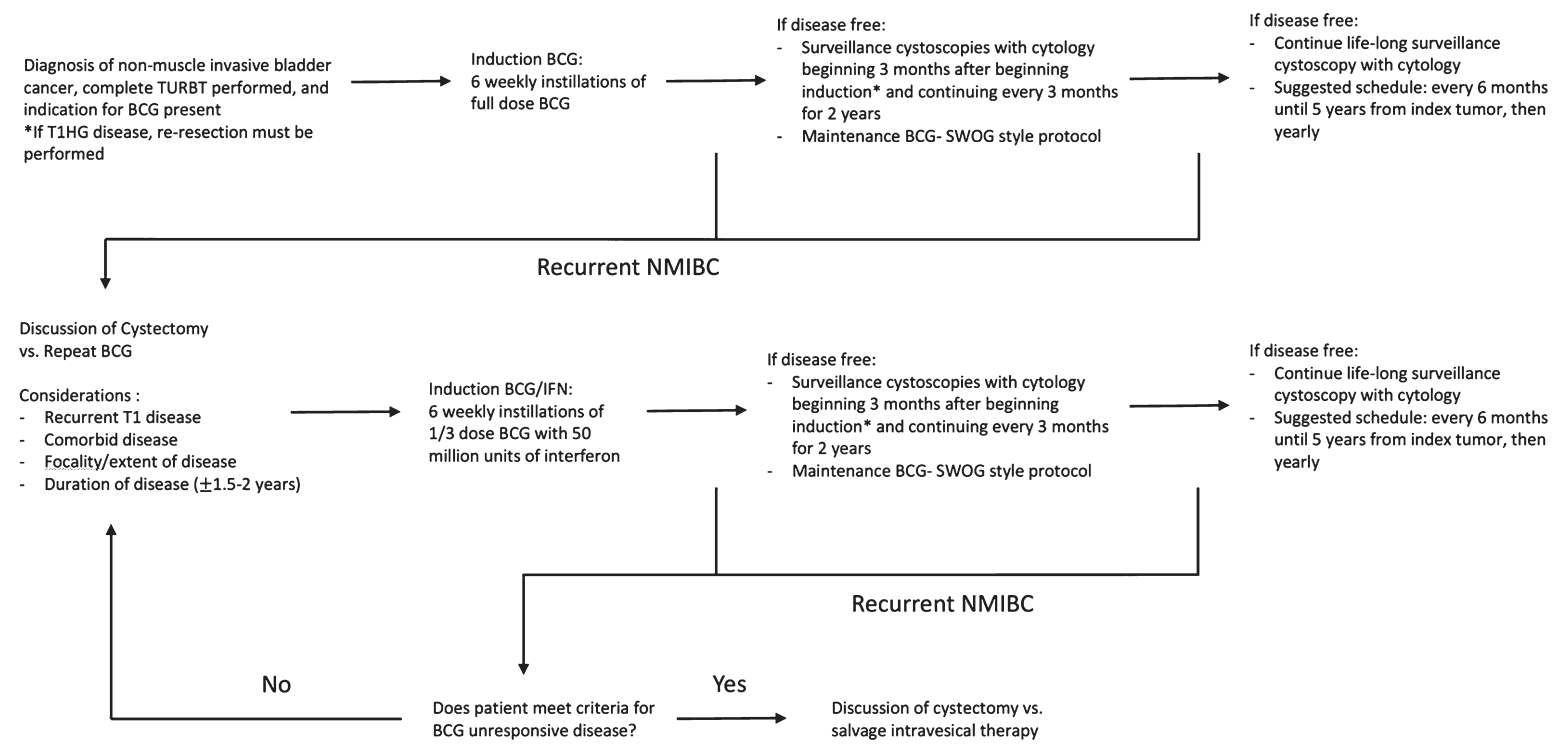

\begin{abstract}
*Consider performing restaging procedure for first surveillance cystoscopy, especially in patients with prior BCG failures, as this has been shown to detect up to $25 \%$ of recurrent disease which would not have been found on pure cystoscopy with cytology (Lightfoot et al. Role of routine transurethral biopsy and isolated upper tract cytology after intravesical treatment of high-grade non-muscle invasive bladder cancer. Int J Urol. (2012); 19(11): 988-993.)
\end{abstract}

Fig. 3. Proposed treatment algorithm for patients with non-muscle invasive bladder cancer.

As previously written by Herr et al. [15], appropriate and consistent nomenclature is crucial not only to avoid unnecessary and ineffective treatments, but also to standardize the interpretation of results for patients who go on to salvage intravesical therapies. Alternative treatment regimens, including intravesical chemotherapy [24-27], device-assisted therapies (i.e. electromotive [28], hyperthermic [29]), and, most recently, mycobacterial cell wall components [30] have been investigated, but comparing study results is nearly impossible given the variable disease definitions used for each study.

In May 2013, a workshop of bladder cancer experts was convened by the AUA and Food and Drug Administration (FDA) to draft guidelines for the designing clinical trials for new NMIBC therapies. Within these guidelines, BCG refractory disease was defined as "patients who received 2 induction courses of BCG, induction plus maintenance (usually within 6 months), or were intolerant of BCG" [31]. It appears prudent that all authors studying intravesical therapy or outcomes after BCG should adopt a single data-driven definition for simplicity, terminology standardization, and to allow for inter-study comparisons. Last, while BCG intolerant patients may be recruited into future studies, we strongly suggest that this cohort be analyzed separately given that: (1) they represent a "pseudo-BCG-naïve" cohort, (2) study intervention is administered earlier in their disease course, and (3) the true effect of BCG on their disease remains unknown.

Limitations of this study include that the initial study was not designed to answer this specific question, the small subgroup size (i.e. T1, multifocal subpopulations) which limited the generation of reliable recurrence-free estimates in instances, and the lack of data regarding disease progression on final analysis. Further study of larger databases or future studies is critical to honing the definition for BCG unresponsive disease. Some may feel that utilization of $1 / 3$ dose $\mathrm{BCG}$ represents undertreatment. Though, there has been good evidence that $1 / 3$ dose BCG, as compared to full dose, is similarly effective and with a lower side effect profile in BCG naïve patients [32, 33]. To date, there have been no reports of reduced dose BCG used to treat BCG failure patients. Further, some consider the addition of IFN to BCG to provide no benefit over repeat BCG monotherapy. This has been shown to be true in BCG naïve patients in a randomized trial [6]. While no direct comparative analysis has been done in prior BCG failure, the 2-year recurrence free survival for BCG/IFN has been shown to be $45 \%$ in a single arm trial [18], while BCG monotherapy has shown a 35\% 2-year 
disease free rate in a pooled analysis and prospective trial $[10,11]$. While superiority cannot be proven, the use of interferon in combination with BCG at least appears to be non-inferior to BCG monotherapy in BCG failure patients. Thus, $1 / 3$ dose BCG does not appear to be undertreatment, at least when used in conjunction with IFN. Given this, the use of the BCG/IFN database for this type of analysis is reasonable. This is the largest data-driven study to date trying to elucidate which demographic and clinicopathologic factors portend the worst response to repeat BCG-based therapy and allows for a more accurate definition of BCG unresponsive disease to be formed.

In conclusion, BCG failure patients who have failed 2 courses of BCG with either persistent or recurrent multifocal papillary disease within 6 months or persistent or recurrent CIS within 12 months of the most recent prior BCG induction course should be considered BCG unresponsive. All other failures should be considered BCG relapsing.

\section{ACKNOWLEDGMENTS}

None.

\section{CONFLICT OF INTEREST}

The authors have no conflict of interest to report.

\section{REFERENCES}

[1] Brosman SA. Experience with bacillus Calmette-Guerin in patients with superficial bladder carcinoma. J Urol 1982;128:27-30.

[2] Lamm DL, Blumenstein BA, Crawford ED, Montie JE, Scardino P, Grossman HB, Stanisic TH, Smith JA Jr, Sullivan J, Sarosdy MF, Crissman A, Coltman CA. A randomized trial of intravesical doxorubicin and immunotherapy with bacille Calmette-Guerin for transitional-cell carcinoma of the bladder. New Engl J Med 199124;325:1205-9.

[3] Lamm DL, Blumenstein BA, David Crawford E, Crissman JD, Lowe BA, Smith JA Jr, Sarosdy MF, Schellhammer PF, Sagalowsky AI, Messing EM, Loehrer P, Grossman HB. Randomized intergroup comparison of bacillus calmetteguerin immunotherapy and mitomycin $\mathrm{C}$ chemotherapy prophylaxis in superficial transitional cell carcinoma of the bladder a southwest oncology group study. Urol Oncol 1995;1:119-26.

[4] Sylvester RJ, Brausi MA, Kirkels WJ, Hoeltl W, Calais Da Silva F, Powell PH, Prescott S, Kirkali Z, van de Beek C, Gorlia T, de Reijke TM; EORTC Genito-Urinary Tract Cancer Group. Long-term efficacy results of EORTC genito-urinary group randomized phase 3 study 30911 comparing intravesical instillations of epirubicin, bacillus
Calmette-Guerin, and bacillus Calmette-Guerin plus isoniazid in patients with intermediate- and high-risk stage Ta T1 urothelial carcinoma of the bladder. Eur Urol 2010;57:76673.

[5] Sylvester RJ, van der Meijden AP, Witjes JA, Kurth K. Bacillus calmette-guerin versus chemotherapy for the intravesical treatment of patients with carcinoma in situ of the bladder: A meta-analysis of the published results of randomized clinical trials. J Urol 2005;174:86-92.

[6] Nepple KG, Lightfoot AJ, Rosevear HM, O’Donnell MA, Lamm DL; Bladder Cancer Genitourinary Oncology Study G. Bacillus Calmette-Guerin with or without interferon alpha- $2 b$ and megadose versus recommended daily allowance vitamins during induction and maintenance intravesical treatment of nonmuscle invasive bladder cancer. J Urol 2010;184:1915-9.

[7] Hall MC, Chang SS, Dalbagni G, Pruthi RS, Seigne JD, Skinner EC, Wolf JS Jr, Schellhammer PF. Guideline for the management of nonmuscle invasive bladder cancer (stages Ta, T1, and Tis): 2007 update. J Urol 2007;178:2314-30.

[8] Babjuk M, Burger M, Zigeuner R, Shariat SF, van Rhijn BW, Compérat E, Sylvester RJ, Kaasinen E, Böhle A, Palou Redorta J, Rouprêt M; European Association of Urology. EAU guidelines on non-muscle-invasive urothelial carcinoma of the bladder: Update 2013. Eur Urol 2013;64:639-53.

[9] Stein JP, Lieskovsky G, Cote R, Groshen S, Feng AC, Boyd S, Skinner E, Bochner B, Thangathurai D, Mikhail M, Raghavan D, Skinner DG. Radical cystectomy in the treatment of invasive bladder cancer: Long-term results in 1,054 patients. J Clin Oncol 2001;19:666-75.

[10] Catalona WJ, Hudson MA, Gillen DP, Andriole GL, Ratliff TL. Risks and benefits of repeated courses of intravesical bacillus Calmette-Guerin therapy for superficial bladder cancer. J Urol 1987;137:220-4.

[11] O'Donnell MA, Boehle A. Treatment options for BCG failures. World J Urol 2006;24:481-7.

[12] Glashan RW. A randomized controlled study of intravesical alpha-2b-interferon in carcinoma in situ of the bladder. J Urol 1990;144:658-61.

[13] Klein EA, Rogatko A, Herr HW. Management of local bacillus Calmette-Guerin failures in superficial bladder cancer. J Urol 1992;147:601-5.

[14] Sarosdy MF, Manyak MJ, Sagalowsky AI, Belldegrun A, Benson MC, Bihrle W, Carroll PR, Ellis WJ, Hudson MA, Sharkey FE. Oral bropirimine immunotherapy of bladder carcinoma in situ after prior intravesical bacille CalmetteGuerin. Urology 1998;51:226-31.

[15] Herr HW, Dalbagni G. Defining bacillus CalmetteGuerin refractory superficial bladder tumors. J Urol 2003;169:1706-8.

[16] Nieder AM, Brausi M, Lamm D, O’Donnell M, Tomita $\mathrm{K}$, Woo H, Jewett MA. Management of stage T1 tumors of the bladder: International Consensus Panel. Urology 2005;66:108-25.

[17] Lerner SP, Dinney C, Kamat A, Bivalacqua TJ, Nielsen M, O'Donnell M, Schoenberg MP, Steinberg G. Clarification of bladder cancer disease sates following treatment of patients with intravesical BCG. Bladder Cancer 2015;1:29-30.

[18] Joudi FN, Smith BJ, O'Donnell MA; National BCGInterferon Phase 2 Investigator Group. Final results from a national multicenter phase II trial of combination bacillus Calmette-Guerin plus interferon alpha-2B for reducing recurrence of superficial bladder cancer. Urol Oncol 2006;24:344-8. 
[19] Lee R, Droller MJ. The natural history of bladder cancer. Implications for therapy. Urol Clin North Am 2000;27:1-13.

[20] O'Donnell MA, Lilli K, Leopold C; National Bacillus Calmette-Guerin/Interferon Phase 2 Investigator G. Interim results from a national multicenter phase II trial of combination bacillus Calmette-Guerin plus interferon alfa- $2 \mathrm{~b}$ for superficial bladder cancer. J Urol 2004;172:888-93.

[21] Merz VW, Marth D, Kraft R, Ackermann DK, Zingg EJ, Studer UE. Analysis of early failures after intravesical instillation therapy with bacille Calmette-Guerin for carcinoma in situ of the bladder. Br J Urol 1995;75:180-4.

[22] Sylvester RJ, van der Meijden AP, Oosterlinck W, Witjes JA, Bouffioux C, Denis L, Newling DW, Kurth K. Predicting recurrence and progression in individual patients with stage Ta T1 bladder cancer using EORTC risk tables: A combined analysis of 2596 patients from seven EORTC trials. Eur Urol 2006;49:466-5;75-7.

[23] Raj GV, Herr H, Serio AM, Donat SM, Bochner BH, Vickers AJ, Bochner BH, Herr H, Donat SM. Treatment paradigm shift may improve survival of patients with high risk superficial bladder cancer. J Urol 2007;177:1283-6.

[24] Barlow LJ, McKiernan JM, Benson MC. Long-term survival outcomes with intravesical docetaxel for recurrent nonmuscle invasive bladder cancer after previous bacillus Calmette-Guerin therapy. J Urol 2013;189:834-9.

[25] Dinney CP, Greenberg RE, Steinberg GD. Intravesical valrubicin in patients with bladder carcinoma in situ and contraindication to or failure after bacillus Calmette-Guerin. Urol Oncol 2013;31:1635-42.

[26] Lightfoot AJ, Breyer BN, Rosevear HM, Erickson BA, Konety BR, O'Donnell MA. Multi-institutional analysis of sequential intravesical gemcitabine and mitomycin $\mathrm{C}$ chemotherapy for non-muscle invasive bladder cancer. Urol Oncol 2014;32:35 e15-9.

[27] Skinner EC, Goldman B, Sakr WA, Petrylak DP, Lenz HJ, Lee CT, Wilson SS, Benson M, Lerner SP, Tangen CM, Thompson IM. SWOG S0353: Phase II trial of intravesical gemcitabine in patients with nonmuscle invasive bladder cancer and recurrence after 2 prior courses of intravesical bacillus Calmette-Guerin. J Urol 2013;190:1200-4.
[28] Di Stasi SM, Giannantoni A, Stephen RL, Capelli G, Navarra P, Massoud R, Vespasiani G. Intravesical electromotive mitomycin $\mathrm{C}$ versus passive transport mitomycin $\mathrm{C}$ for high risk superficial bladder cancer: A prospective randomized study. J Urol 2003;170:777-82.

[29] Gofrit ON, Shapiro A, Pode D, Sidi A, Nativ O, Leib Z, Witjes JA, van der Heijden AG, Naspro R, Colombo R. Combined local bladder hyperthermia and intravesical chemotherapy for the treatment of high-grade superficial bladder cancer. Urology 2004;63:466-71.

[30] Morales A, Herr H, Steinberg G, Given R, Cohen Z, Amrhein J, et al. Efficacy and safety of MCNA in patients with non-muscle invasive bladder cancer at high-risk of recurrence and progression who have failed treatment with bacillus Calmette-Guerin. J Urol 2015;193:1135-43.

[31] Jarow JP, Lerner SP, Kluetz PG, Liu K, Sridhara R, Bajorin D, Chang S, Dinney CP, Groshen S, Morton RA, O’Donnell MA, Quale DZ, Schoenberg M, Seigne J, Vikram B. Clinical trial design for the development of new therapies for nonmuscle-invasive bladder cancer: Report of a Food and Drug Administration and American Urological Association public workshop. Urology 2014;83:262-4.

[32] Martínez-Piñeiro JA, Martínez-Piñeiro L, Solsona E, Rodríguez RH, Gómez JM, Martín MG, Molina JR, Collado AG, Flores N, Isorna S, Pertusa C, Rabadán M, Astobieta A, Camacho JE, Arribas S, Madero R; Club Urológico Español de Tratamiento Oncológico (CUETO). Has a 3fold decreased dose of bacillus Calmette-Guerin the same efficacy against recurrences and progression of T1G3 and Tis bladder tumors than the standard dose? Results of a prospective randomized trial. J Urol 2005;174:1242-7.

[33] Oddens J, Brausi M, Sylvester R, Bono A, van de Beek C, van Andel G, Gontero P, Hoeltl W, Turkeri L, Marreaud S, Collette S, Oosterlinck W. Final results of an EORTC-GU cancers group randomized study of maintenance bacillus Calmette-Guerin in intermediate- and high-risk Ta, T1 papillary carcinoma of the urinary bladder: One-third dose versus full dose and 1 year versus 3 years of maintenance. Eur Urol 2013;63:462-72. 